\title{
Gehstrecke verlängert, Krankheitsprogression gebremst, Lebensqualität verbessert
}

- Zwar ist eine Heilung bei pulmonal-arterieller Hypertonie (PAH) auch in absehbarer Zukunft wohl nicht möglich, es gibt aber durchaus Fortschritte bei der Behandlung. „Das dürfte sich auch künftig weiter fortsetzen“, so Prof. Ardeschir Ghofrani, Gießen. Es werde zu verschiedenen neuen Therapieoptionen geforscht, wobei große Hoffnungen auf die löslichen GuanylatcyclaseStimulatoren gesetzt werden.

Doch auch bei den bereits etablierten Therapieprinzipien wie den Phosphodiesterase (PDE-)5-Hemmern stehen nach Prof. Nazzareno Galiè, Bologna, Fortschritte ins Haus. Denn auch für Tadalafil wurde eine gute kli- nische Wirksamkeit bei der PAH belegt. So hat die Studie PHIRST (Pulmonary Arterial Hypertension and Responses to Tadalafil) eindeutig gezeigt, dass die Substanz die körperliche Belastbarkeit bei PAH steigert. In den USA ist sie bereits bei PAH zugelassen. In der Studie wurden 405 PAH-Patienten, die zum Teil auch Bosentan erhielten, 16 Wochen lang kontrolliert mit vier Dosen Tadalafil (2,5 mg, $10 \mathrm{mg}$, $20 \mathrm{mg}$ oder $40 \mathrm{mg}$ einmal täglich) behandelt. Ihre Gehstrecke im 6-Minuten-Gehtest verlängerte sich deutlich, wobei der Effekt dosisabhängig war und unter 40 mg Tadalafil eine statistisch signifikante Besserung resultierte. Der PDE-5-Hemmer verlängerte laut Galiè außerdem die Zeit bis zur klinischen Verschlechterung der PAH, wobei dies bei Patienten mit Tadalafil generell seltener geschah. „Relevant ist auch die in der Studie registrierte deutliche Besserung der gesundheitsbezogenen Lebensqualität mit dem PDE-5-Hemmer“, sagte Galiè. In Subgruppenanalysen wurde nach seinen Angaben außerdem eine Verbesserung hämodynamischer Parameter registriert, insbesondere beim pulmonal-arteriellen Druck und beim Herzindex. „Die Therapieeffekte waren in etwa denen vergleichbar, die wir mit der etablierten Therapie bei der $\mathrm{PAH}$ sehen“, sagte Galiè. Tadalafil sei zudem gut verträglich, als häufigste Nebenwirkungen traten unter der Therapie Kopfschmerzen, Myalgien und Flush auf.

Symposium bei der 19. Jahrestagung der ERS, 16.9.2009 in Wien, Veranstalter: Lilly

\section{Chronische Bronchitis}

\section{Sekretolyse plus Atemtherapie für effektive Symptombekämpfung}

\begin{abstract}
- Patienten mit chronischer Bronchitis kann mit einer Kombination aus medikamentöser Therapie und Atemphysiotherapie effektiv geholfen werden. Die Atemwege werden geöffnet, der zähe Schleim wird dünnflüssiger und kann so leichter abgehustet werden. Den Erfolg einer derartigen Kombinationsbehandlung bestätigt z.B. der Fallbericht eines Patienten, der nach einem Schädel-Hirn- und Thoraxtrauma an einer chronischen Bronchitis erkrankt ist.

Chronische Bronchitis ist durch chronischen Husten und vermehrte Schleimbildung gekennzeichnet. Laut WHO kann die Diagnose gestellt werden, wenn bei einem Patienten in zwei aufeinanderfolgenden Jahren über mindestens drei Monate pro Jahr Husten und Auswurf bestanden haben. In Deutschland sind 10-15\% der Erwachsenen von dieser Atemwegserkrankung betroffen.

Einer dieser Betroffenen ist ein Patient nach Schädel-Hirn- und Thoraxtrauma. Bei der körperlichen Untersuchung fallen bronchitische Rasselgeräusche wechselnder Intensität auf. Insgesamt macht er einen verlangsamten und antriebsarmen Eindruck. Eine Lungenfunktionsdiagnostik
\end{abstract}

ist nicht durchführbar. Bei Exazerbationen sind im Röntgenbild der Lunge Infiltrate zu erkennen. Laborchemisch fallen erhöhte CRP- und Leukozytenwerte auf.

Der Patient wird symptomatisch medikamentös und physiotherapeutisch behandelt. Als Dauermedikation hat sich die Gabe von GeloMyrtol ${ }^{\circledR}$ forte als ein gut verträgliches Medikament bewährt. Bei Exazerbationen erfolgt eine Dosissteigerung dieser Dauermedikation auf dreimal eine Kapsel täglich. Zusätzlich inhaliert er im Winterhalbjahr und wendet das Atemtherapiegerät GeloMuc ${ }^{\circledR}$ an. Unter der Kombination von medikamentöser Sekretolyse mit Myrtol standardisiert und der Anwendung des physiotherapeutischen Atemtherapiegeräts kam es in der vergangenen und der aktuellen Saison deutlich seltener zu Exazerbationen der Bronchitis, und Antibiotika waren seltener nötig. Mithilfe des Atemtherapiegerätes wurde das morgendliche Bronchialsekret dünnflüssiger, und der Patient konnte es so noch besser abhusten (verbesserte Bronchialtoilette).

red

Pohl Boskamp

\section{kurz gemeldet}

\section{Mit der Asthmatherapie kleinste Atemwege erreichen}

Eine Asthmatherapie soll die Entzündung hemmen sowie die bronchiale Hyperreagibilität und die Obstruktion vermindern. Inuvair ${ }^{\circledR}$ Mikrosol bei moderatem bis schwerem persistierendem Asthma erreicht dies durch die Wirkstoffe Beclometason und Formoterol. Mit der Sprühwolke aus extrafeinen Partikeln gelangen die Wirkstoffe bis in kleine Atemwege. Hier scheint die Entzündung stärker zu sein als in den großen Bronchien. Die Partikel des Kombipräparates haben einen massenaerodynamischen Durchmesser (MMAD) von ca. 1,5 $\mu \mathrm{m}$. Nur Partikel mit MMAD von 1-2 $\mu \mathrm{m}$ erreichen überwiegend die Lungenperipherie. Der positive Effekt von extrafeinem Beclometason bei schlecht kontrolliertem Asthma wurde in einer Studie untersucht [1]. Patienten erhielten zusätzlich zur bisherigen Therapie Beclometason in extrafeiner Formulierung oder Fluticason. Mit Beclometason besserten sich die Atemstromwerte FEF25-75\%, FEV 1 und PEF signifikant. Dabei zeigte sich v.a. an der bessereren FEF25-75\% die Erweiterung der kleinen Bronchien, und der Bedarf an spasmolytischen Notfallpräparaten sank. red

1. Thongngarm T et al., Asthma 2005; 42:257-63. Janssen Cilag 